\title{
A CLASS OF UNIVALENT FUNCTIONS WITH REAL COEFFICIENTS
}

\author{
MILUTIN OBRADOVIĆ AND NIKOLA TUNESKI
}

\begin{abstract}
In this paper we study class $\mathcal{S}^{+}$of univalent functions $f$ such that $\frac{z}{f(z)}$ has real and positive coefficients. For such functions we give estimates of the Fekete-Szegó functional and sharp estimates of their initial coefficients and logarithmic coefficients. Also, we present necessary and sufficient conditions for $f \in \mathcal{S}^{+}$to be starlike of order $1 / 2$.
\end{abstract}

\section{INTRODUCTION}

Let $\mathcal{A}$ be the class of functions $f$ that are analytic in the open unit disc $\mathbb{D}=\{z:|z|<1\}$ of the form $f(z)=z+a_{2} z^{2}+a_{3} z^{3}+\cdots$. Then the class of starlike functions of order $\alpha, 0 \leq \alpha<1$, is defined by

$$
\mathcal{S}^{*}(\alpha)=\left\{f \in \mathcal{A}: \operatorname{Re} \frac{z f^{\prime}(z)}{f(z)}>\alpha, z \in \mathbb{D}\right\},
$$

while $\mathcal{S}^{*} \equiv \mathcal{S}^{*}(0)$ is the well known class of starlike functions mapping the unit disc onto a starlike region $D$, i.e.,

$$
w \in f(D) \quad \Leftrightarrow \quad t w \in f(\mathbb{D}) \text { for all } t \in[0,1] .
$$

More on this classes can be found in [7] and [1.

Further, let $\mathcal{S}^{+}$denote the class of univalent functions in the unit disc with the next representation

$$
\frac{z}{f(z)}=1+b_{1} z+b_{2} z^{2}+\ldots, \quad b_{n} \geq 0, n=1,2,3, \ldots
$$

For example, the Silverman class (the class with negative coefficients) is included in the class $\mathcal{S}^{+}$. Namely, that class consists of univalent functions of the form

$$
f(z)=z-a_{2} z^{2}-a_{3} z^{3}-\ldots, a_{n} \geq 0, n=2,3, \ldots
$$

which implies that

$$
\frac{z}{f(z)}=\frac{1}{1-a_{2} z-a_{3} z^{2}-\cdots}
$$

i.e., $\frac{z}{f(z)}$ has the form (11). Also, the Koebe function $k(z)=\frac{z}{(1+z)^{2}} \in \mathcal{S}^{+}$. The next characterization is valid for the class $\mathcal{S}^{+}$(see [2]):

$$
f \in \mathcal{S}^{+} \quad \Leftrightarrow \quad \sum_{n=2}^{\infty}(n-1) b_{n} \leq 1
$$

From the relations (10) and (2) we have that

$$
b_{2}+2 b_{3} \leq 1 \quad\left(\Rightarrow \quad 0 \leq b_{2} \leq 1, \quad 0 \leq b_{3} \leq 1 / 2\right) .
$$

2000 Mathematics Subject Classification. 30C45, 30C50, 30C55.

Key words and phrases. univalent, real coefficients, Fekete-Szegö, logarithmic coefficients, coefficient estimates. 
If we put $f(z)=z+a_{2} z^{2}+\ldots$, then by using (10) we easily obtain that

$$
b_{1}=-a_{2}, \quad b_{2}=a_{2}^{2}-a_{3} .
$$

This implies that $0 \leq b_{1} \leq 2$. From (1) we obtain

$$
\log \frac{f(z)}{z}=-\log \left(1+b_{1} z+b_{2} z^{2}+\cdots\right),
$$

or

$$
\sum_{n=1}^{\infty} 2 \gamma_{n} z^{n}=-b_{1} z+\left(\frac{1}{2} b_{1}^{2}-b_{2}\right) z^{2}+\left(-\frac{1}{3} b_{1}^{3}+b_{1} b_{2}-b_{3}\right) z^{3}+\cdots .
$$

(We call $\gamma_{n}, n=1,2, \ldots$ the logarithmic coefficients of the function $f$.) From the last relation we have

$$
\left\{\begin{array}{l}
2 \gamma_{1}=-b_{1} \\
2 \gamma_{2}=\frac{1}{2} b_{1}^{2}-b_{2} \\
2 \gamma_{3}=-\frac{1}{3} b_{1}^{3}+b_{1} b_{2}-b_{3}
\end{array}\right.
$$

For functions $f$ in $\mathcal{S}^{+}$we give sharp estimates of their logarithmic coefficients $\gamma_{1}, \gamma_{2}$ and $\gamma_{3}$ of $f$ and lower and upper bound of the Fekete-Szegö functional $\left(a_{3}-\gamma a_{2}^{2}\right)$. Additionally, sharp estimates of coefficients $a_{2}, a_{3}, a_{4}$ and $a_{5}$ for functions in a class containing $\mathcal{S}^{+}$is given. At the end the relation between the class $\mathcal{S}^{+}$and the class of starlike functions is studied.

\section{Results OVER the COEFFicients}

We start the section with a study of the Fekete-Szegő functional for the functions in the class $\mathcal{S}^{+}$

Theorem 1. For each $f \in \mathcal{S}^{+}$we have

$$
-1 \leq a_{3}-\gamma a_{2}^{2} \leq \begin{cases}1+2 e^{-2 \gamma /(1-\gamma)}, & 0 \leq \gamma \leq \frac{\nu_{0}}{1+\nu_{0}}=0.456278 \ldots \\ 2(1-\gamma) \frac{\left(\nu_{0}+1\right)^{2}}{2 \nu_{0}+1}, & \frac{\nu_{0}}{1+\nu_{0}} \leq \gamma<1 .\end{cases}
$$

where $\nu_{0}=0.83927 \ldots$ is the positive real root of the equation

$$
2(2 \nu+1) e^{-2 \nu}=1 .
$$

The lower bound is sharp due to the function $f_{1}(z)=\frac{z}{1+z^{2}}$.

Proof. We will use the same method as in the proof of Fekete-Szego theorem for the class $\mathcal{S}$ (see [1, Theorem 3.8, p. 104]). First, from the relation (4) we have that

$$
-1 \leq a_{3}-a_{2}^{2}=-b_{2} \leq 0 .
$$

Since $a_{2}$ and $a_{3}$ are real we can put (as in that proof) $a_{2}=-2 \int_{0}^{\infty} \varphi(t) d t$, where $\varphi$ is real function and $|\varphi(t)| \leq e^{-t}$. If we put

$$
\int_{0}^{\infty}[\varphi(t)]^{2} d t=\left(\nu+\frac{1}{2}\right) e^{-2 \nu}, \quad 0 \leq \lambda<\infty,
$$

then by Valiron-Landau lemma we have that $\left|a_{2}\right| \leq 2(\nu+1) e^{-\nu}$. Also, we have (as [1]):

$$
a_{3}-a_{2}^{2}=1-4 \int_{0}^{\infty}[\varphi(t)]^{2} d t=1-4\left(\nu+\frac{1}{2}\right) e^{-2 \nu} \leq 0
$$

if and only if $0 \leq \nu \leq \nu_{0}$, where $\nu_{0}=0.83927 \ldots$ is the root of the equation (6). 
Now, for $0 \leq \gamma<1$ and for $0 \leq \nu \leq \nu_{0}$ we have that

$$
\begin{aligned}
a_{3}-\gamma a_{2}^{2} & \leq 4(1-\gamma)\left(\int_{0}^{\infty} \varphi(t) d t\right)^{2}-4 \int_{0}^{\infty}[\varphi(t)]^{2} d t+1 \\
& =4 e^{-2 \nu}\left[(1-\gamma)(\nu+1)^{2}-\left(\nu+\frac{1}{2}\right)\right]+1 \\
& =: \psi(\nu) .
\end{aligned}
$$

It is an elementary fact that the function $\psi$ has its maximum $\psi(\gamma /(1-\gamma))$ if $\frac{\gamma}{1-\gamma} \in\left[0, \nu_{0}\right]$ and $\psi\left(\nu_{0}\right)$ if $\frac{\gamma}{1-\gamma} \notin\left[0, \nu_{0}\right]$, which gives the right estimation in the theorem (in the second case we used that $\nu_{0}$ satisfies the equation (6) ).

On the other hand side $a_{3}-\gamma a_{2}^{2} \geq a_{3}-a_{2}^{2} \geq-1$.

Next we give sharp estimates of the first three logarithmic coefficients for functions in $\mathcal{S}^{+}$.

Theorem 2. Let $f \in \mathcal{S}^{+}$and let $\gamma_{1}, \gamma_{2}, \gamma_{3}$ be its logarithmic coefficients. Then

(a) $-1 \leq \gamma_{1} \leq 0$;

(b) $-\frac{1}{2} \leq \gamma_{2} \leq \frac{\left(\nu_{0}+1\right)^{2}}{2\left(2 \nu_{0}+1\right)}=0.631464 \ldots$,

where $\nu_{0}=0.83927 \ldots$ is the solution of the equation ([6);

(c) $-\frac{1}{4} \leq \gamma_{3} \leq \frac{1}{3}$

Some of these results are the best possible.

Proof.

(a) It is evident since $\gamma_{1}=-\frac{1}{2} b_{1}$ (from (5)) and $0 \leq b_{1} \leq 2$. The functions $f_{1}(z)=\frac{z}{1+z^{2}}$ and $f_{2}(z)=\frac{z}{(1+z)^{2}}$ show that the result is the best possible.

(b) From (4) and (5) we have that

$$
\gamma_{2}=\frac{1}{2}\left(\frac{1}{2} b_{1}^{2}-b_{2}\right)=\frac{1}{2}\left(a_{3}-\frac{1}{2} a_{2}^{2}\right)
$$

and the result directly follows from Theorem 1 for $\gamma=\frac{1}{2}$. For the function $f_{1}(z)=\frac{z}{1+z^{2}}$ we have that $\log \frac{f_{1}(z)}{z}=-\log \left(1+z^{2}\right)=-z^{2}+\ldots$, which means that left hand side estimate is the best possible.

We were not able to prove sharpness of the right hand side of the inequality (the upper bound of $\gamma_{2}$ ), but it is worth pointing that the estimate goes in a line with the sharp estimate coresponding to the univalent functions, known to be (see [1, Theorem 3.8] or [7, p.136])

$$
\left|\gamma_{2}\right| \leq \frac{1}{2}\left(1+2 e^{-2}\right)=0.635 \ldots
$$

(c) From (5) we have

$$
2 \gamma_{3}=-\frac{1}{3} b_{1}^{3}+b_{1} b_{2}-b_{3}=: u\left(b_{1}\right),
$$

where

$$
u(t)=-\frac{1}{3} t^{3}+b_{2} t-b_{3}, \quad 0 \leq t \leq 2 .
$$

Since $u^{\prime}(t)=-t^{2}+b_{2}$ and $u^{\prime}(t)=0$ for $t_{0}=\sqrt{b_{2}}$, then the function $u$ attains its maximum

$$
u\left(t_{0}\right)=u\left(\sqrt{b_{2}}\right)=\frac{2}{3} b_{2}^{3 / 2}-b_{3} \leq \frac{2}{3}\left(1-2 b_{3}\right)^{3 / 2}-b_{3} \leq \frac{2}{3},
$$


because $b_{2} \leq 1-2 b_{3}$ (see (3) ) and the last function is a decreasing function of $b_{3}, 0 \leq b_{3} \leq \frac{1}{2}$. This provides that $\gamma_{3} \leq \frac{1}{3}$. For the function $f_{3}(z)=\frac{z}{1+z+z^{2}}$ we have

$$
\log \frac{f_{3}(z)}{z}=-\log \left(1+z+z^{2}\right)=-z-\frac{1}{2} z^{2}+\frac{2}{3} z^{3}+\cdots,
$$

i.e. $\gamma_{3}=\frac{1}{3}$.

As for lower bound for $\gamma_{3}$, by using (5) and (4), we have

$$
\begin{aligned}
-2 \gamma_{3} & =\frac{1}{3} b_{1}^{3}-b_{1} b_{2}+b_{3} \\
& =\frac{1}{3} b_{1}^{3}-b_{1}\left(b_{1}^{2}-a_{3}\right)+b_{3} \\
& =-\frac{2}{3} b_{1}^{3}+a_{3} b_{1}+b_{3} \\
& =v\left(b_{1}\right),
\end{aligned}
$$

where

$$
v(t)=-\frac{2}{3} t^{3}+a_{3} t+b_{3}, \quad(0 \leq t \leq 2)
$$

From here we have

$$
v^{\prime}(t)=-2 t^{2}+a_{3} .
$$

If $a_{3} \leq 0$, then $v^{\prime}(t) \leq 0$, and if $a_{3}>0$ then we can write

$$
v^{\prime}(t)=-2\left(b_{1}^{2}-a_{3}\right)-a_{3}=-2 b_{2}-a_{3}
$$

and also we have $v^{\prime}(t)<0$, since $0 \leq b_{2} \leq 1$. It means that the function $v$ is a decreasing function, which gives that

$$
-2 \gamma_{3} \leq v(0)=b_{3} \leq \frac{1}{2}
$$

i.e $\gamma_{3} \geq-\frac{1}{4}$. The function $f_{4}(z)=\frac{z}{1+z^{3} / 2}$ shows that the result is the best possible.

Let $\mathcal{U}(\lambda), 0<\lambda \leq 1$, denote the class of functions $f \in \mathcal{A}$ which satisfy the condition

$$
\left|\left(\frac{z}{f(z)}\right)^{2} f^{\prime}(z)-1\right|<\lambda \quad(z \in \mathbb{D}) .
$$

We put $\mathcal{U}(1) \equiv \mathcal{U}$. More about classes $\mathcal{U}$ and $\mathcal{U}(\lambda)$ we can find in [5, [7, 3] and [4].

Let $\mathcal{U}^{+}(\lambda), 0<\lambda \leq 1$, denote the class of functions $f$ satisfy the conditions (11) and (7). By using (2) we can conclude that $\mathcal{U}^{+}(1) \equiv \mathcal{S}^{+}$. For example, the function

$$
\begin{aligned}
f_{\lambda}(z) & =\frac{z}{1+(1+\lambda) z+\lambda z^{2}} \\
& =z-(1+\lambda) z^{2}+\left(1+\lambda+\lambda^{2}\right) z^{3}-\left(1+\lambda+\lambda^{2}+\lambda^{3}\right) z^{4}+\cdots .
\end{aligned}
$$

belongs to the class $\mathcal{U}^{+}(\lambda)$ and it is extremal in many cases.

Also, if $f \in \mathcal{U}^{+}(\lambda)$ and has the form (11) then by (2):

$$
\sum_{n=2}^{\infty}(n-1) b_{n} \leq \lambda,
$$


which implies the appropriate inequalites :

$$
0 \leq b_{2} \leq \lambda, \quad b_{2}+2 b_{3} \leq \lambda, \quad b_{2}+2 b_{3}+3 b_{4} \leq \lambda, \ldots
$$

For the coefficients of functions from the class $\mathcal{U}^{+}(\lambda)$, the next theorem is valid.

Theorem 3. If $f(z)=z+a_{2} z^{2}+a_{3} z^{3}+\cdots$ belongs to the class $\mathcal{U}^{+}(\lambda), 0<\lambda \leq 1$, then we have

$$
\begin{aligned}
-(1+\lambda) & \leq a_{2} \leq 0, \\
-\lambda & \leq a_{3} \leq 1+\lambda+\lambda^{2}, \\
-\left(1+\lambda+\lambda^{2}+\lambda^{4}\right) & \leq a_{4} \leq \frac{4 \lambda}{3} \sqrt{\frac{2 \lambda}{3}}, \\
a_{5} & \geq\left\{\begin{array}{cc}
-\lambda / 3, & 0<\lambda \leq 4 / 27 \\
-9 \lambda^{2} / 4, & 4 / 27 \leq \lambda \leq 1
\end{array} .\right.
\end{aligned}
$$

All these inequalities are sharp.

Proof. For $f(z)=z+a_{2} z^{2}+a_{3} z^{3}+\cdots$ and $f \in \mathcal{U}(\lambda), 0<\lambda \leq 1$ it is shown in [4] the next sharp inequalities:

$$
\left|a_{2}\right| \leq 1+\lambda, \quad\left|a_{3}\right| \leq 1+\lambda+\lambda^{2}, \quad\left|a_{4}\right| \leq 1+\lambda+\lambda^{2}+\lambda^{3} .
$$

In the same paper the authors conjectured that $\left|a_{n}\right| \leq \sum_{k=0}^{n-1} \lambda^{k}$. Since the function $f_{\lambda}$ defined by (8) belongs to the class $\mathcal{U}^{+}(\lambda)$, then the lower bounds for $a_{2}$ and $a_{4}$ and the upper bounds for $a_{3}$ are valid and sharp. We only need to prove the lower bounds for $a_{3}$ and $a_{5}$ and the upper bounds for $a_{2}$ and $a_{4}$.

If $f(z)=z+a_{2} z^{2}+a_{3} z^{3}+\cdots$ and $f$ has the form (1), then by comparing the coefficients we easily conclude that

$$
\left\{\begin{array}{l}
a_{2}=-b_{1}, \\
a_{3}=-b_{2}+b_{1}^{2} \\
a_{4}=-b_{3}+2 b_{1} b_{2}-b_{1}^{3} \\
a_{5}=-b_{4}+b_{2}^{2}+2 b_{1} b_{3}-3 b_{1}^{2} b_{2}+b_{1}^{4} .
\end{array}\right.
$$

From $a_{2}=-b_{1}$ and $b_{1} \geq 0$, we have $a_{2} \leq 0$. Also, by using (9) and (10), we obtain

$$
-a_{3}=b_{2}-b_{1}^{2} \leq b_{2} \leq \lambda,
$$

which implies $a_{3} \geq-\lambda$. The function $f_{6}(z)=\frac{z}{1+\lambda z^{2}}\left(=z-\lambda z^{3}+\cdots\right)$ shows that two previous results are the best possible.

Further, from (10) we have

$$
a_{4}=-b_{3}+2 b_{1} b_{2}-b_{1}^{3} \leq 2 b_{2} b_{1}-b_{1}^{3}=: w\left(b_{1}\right),
$$

where $0 \leq b_{1} \leq 1+\lambda$ (since $b_{1}=-a_{2} \leq 1+\lambda$ ). It is an elementary fact to get that the function $w$ has its maximun $\frac{4 b_{2}}{3} \sqrt{\frac{2 b_{2}}{3}}$ for $b_{1}=\sqrt{\frac{2 b_{2}}{3}}$. It means that

$$
a_{4} \leq \frac{4 b_{2}}{3} \sqrt{\frac{2 b_{2}}{3}} \leq \frac{4 \lambda}{3} \sqrt{\frac{2 \lambda}{3}},
$$

since $0 \leq b_{2} \leq \lambda$. The function

$$
f_{7}(z)=\frac{z}{1+\sqrt{\frac{2 \lambda}{3}} z+\lambda z^{2}}
$$

shows that the result is the best possible. 
Finally, from (10) we also have

$$
\begin{aligned}
-a_{5} & =b_{4}-b_{2}^{2}-2 b_{1} b_{3}+3 b_{1}^{2} b_{2}-b_{1}^{4} \\
& \leq b_{4}+3 b_{1}^{2} b_{2}-b_{1}^{4} \\
& \leq \frac{1}{3}\left(\lambda-b_{2}\right)+3 b_{2} b_{1}^{2}-b_{1}^{4} \\
& \leq \frac{9}{4} b_{2}^{2}+\frac{1}{3}\left(\lambda-b_{2}\right) \\
& \leq\left\{\begin{array}{cc}
\lambda / 3, & 0<\lambda \leq 4 / 27 \\
9 \lambda^{2} / 4 & 4 / 27 \leq \lambda \leq 1
\end{array},\right.
\end{aligned}
$$

where we used the relation (9) and the same method as in the previous case. The functions

$$
f_{2}(z)=\frac{z}{1+\sqrt{\frac{3 \lambda}{2}} z+\lambda z^{2}} \quad \text { and } \quad f_{8}(z)=\frac{z}{1+\frac{\lambda}{3} z^{4}}
$$

show that the result is the best possible.

For $\lambda=1$ from the previous theorem we have

Corollary 1. Let $f(z)=z+a_{2} z^{2}+a_{3} z^{3}+\cdots$ belong to the class $\mathcal{S}^{+}$. Then we have the next sharp inequalities

$$
-2 \leq a_{2} \leq 0, \quad-1 \leq a_{3} \leq 3, \quad-4 \leq a_{4} \leq \frac{4}{3} \sqrt{\frac{2}{3}}, \quad-\frac{9}{4} \leq a_{5} \leq 5 .
$$

We note that upper bound for $a_{5}$ follows from de Brange's theorem.

\section{RELATion With STARLike FUnCTIONS}

In this section we study the relation between the class $\mathcal{S}^{+}$and the class of starlike functions.

Theorem 4. Let $f \in \mathcal{A}$ and satisfy the condition (1). Then the condition

$$
\sum_{n=1}^{\infty}(2 n-1) b_{n} \leq 1
$$

is necessary and sufficient for $f$ to be in the class $\mathcal{S}^{\star}(1 / 2)$.

Proof. The sufficient condition follows from the result given in the paper of Reade, Silverman and Todorov [6].

Let's prove the necessary case. If $f \in \mathcal{S}^{\star}\left(\frac{1}{2}\right)$, then

$$
\left|\frac{\frac{z f^{\prime}(z)}{f(z)}-1}{\frac{z f^{\prime}(z)}{f(z)}}\right|<1 \quad(z \in \mathbb{D})
$$

or equivalently

$$
\frac{\left|z\left(\frac{z}{f(z)}\right)^{\prime}\right|}{\left|\frac{z}{f(z)}-z\left(\frac{z}{f(z)}\right)^{\prime}\right|}<1 \quad(z \in \mathbb{D})
$$


and from here

$$
\frac{\left|\sum_{n=1}^{\infty} n b_{n} z^{n}\right|}{\left|1-\sum_{n=2}^{\infty}(n-1) b_{n} z^{n}\right|}<1 \quad(z \in \mathbb{D})
$$

If $z=r(0<r<1)$ we have from the last inequality that

$$
\frac{\sum_{n=1}^{\infty} n b_{n} r^{n}}{1-\sum_{n=2}^{\infty}(n-1) b_{n} r^{n}}<1
$$

which implies the condition

$$
\sum_{n=1}^{\infty}(2 n-1) b_{n} r^{n}<1
$$

Finally, when $r \rightarrow 1$ we have

$$
\sum_{n=1}^{\infty}(2 n-1) b_{n} \leq 1
$$

i.e., the relation (11).

Remark 1. Since the class of convex functions is the subset of the class $S^{\star}(1 / 2)$, then if a function $f$ is convex and

$$
\frac{z}{f(z)}=1+b_{1} z+b_{2} z^{2}+\ldots
$$

with $b_{n} \geq 0$ for $n=1,2, \ldots$, we have

$$
\sum_{n=1}^{\infty}(2 n-1) b_{n} \leq 1
$$

The converse is not true. Namely, for the function

$$
f(z)=\frac{z}{1+\frac{1}{3} z^{2}}
$$

we have that

$$
\frac{z}{f(z)}=1+\frac{1}{3} z^{2}
$$

and

$$
\sum_{n=1}^{\infty}(2 n-1) b_{n}=1
$$

but

$$
1+\frac{z f^{\prime \prime}(z)}{f^{\prime}(z)}=\frac{1-2 z^{2}+\frac{1}{9} z^{4}}{1-\frac{1}{9} z^{4}}<0
$$

for $z=r(0<r<1)$ and $r$ close to 1 .

Theorem 5. Let $f \in \mathcal{S}^{+}$and let $b_{1}=0$, then $f \in \mathcal{S}^{\star}$.

Proof. Since $f \in \mathcal{S}^{+}$, then $\sum_{n=2}^{\infty}(n-1) b_{n} \leq 1$, and since $b_{1}=0$, then also $\sum_{n=2}^{\infty}(n-1) b_{n} \leq 1=$ $1-b_{1}$, which implies, by result of Reade et al. ([6]) (see the previous sited result in Theorem [4), that $f \in \mathcal{S}^{\star}$.

We note that if $b_{1}=0$, then $\operatorname{Re} \frac{f(z)}{z}>\frac{1}{2}(z \in \mathbb{D})$ since

$$
|z / f(z)-1| \leq|z|^{2} \sum_{n=2}^{\infty} b_{n} \leq \sum_{n=2}^{\infty}(n-1) b_{n} \leq|z|^{2}<1 \quad(z \in \mathbb{D}) .
$$


But under the condition of this theorem we do not have that $f \in \mathcal{S}^{\star}(1 / 2)$. For example, for the function $f_{1}(z)=\frac{z}{1+z^{2}}$ we have $b_{1}=0$, but $\sum_{n=1}^{\infty}(2 n-1) b_{n}=3$, which means that $f_{1} \notin \mathcal{S}^{\star}(1 / 2)$ (by the previous theorem).

Theorem 6. Let $f \in \mathcal{S}^{+}$. Then the function

$$
g(z)=z+\frac{1}{2}\left(\frac{z}{f(z)}-1-b_{1} z\right)
$$

is univalent in $\mathbb{D}$. More precisely, $\operatorname{Re} g^{\prime}(z)>0(z \in \mathbb{D}), g \in \mathcal{S}^{\star}$ and $g \in \mathcal{U}$.

Proof. It is well-known that if $f(z)=z+a_{2} z^{2}+a_{3} z^{3}+\cdots$ and $\sum_{n=2}^{\infty} n\left|a_{n}\right| \leq 1$, then $\operatorname{Re} f^{\prime}(z)>0$ $(z \in \mathbb{D})$ and $f \in \mathcal{S}^{\star}$ with $\left|\frac{z f^{\prime}(z)}{f(z)}-1\right|<1(z \in \mathbb{D})$. It is easily to prove those statement (in the second case better to consider the form $\left.\left|z f^{\prime}(z)-f(z)\right|<|f(z)|\right)$.

By (12) we have

$$
g(z)=z+\sum_{n=2}^{\infty} \frac{1}{2} b_{n} z^{n} .
$$

Since $f \in \mathcal{S}^{+}$implies $\sum_{n=2}^{\infty}(n-1) b_{n} \leq 1$ and since $\frac{n}{2(n-1)} \leq 1$ for $n \geq 2$, then

$$
\sum_{n=2}^{\infty} n\left(\frac{1}{2} b_{n}\right)=\sum_{n=2}^{\infty}(n-1) b_{n} \frac{n}{2(n-1)} \leq \sum_{n=2}^{\infty}(n-1) b_{n} \leq 1 .
$$

By previous remarks we have $\operatorname{Re} g^{\prime}(z)>0(z \in \mathbb{D})$ and $g \in \mathcal{S}^{\star}$. Also, $g \in \mathcal{U}$ by the result given in [5].

\section{REFERENCES}

1. Duren P.L., Univalent function, Springer-Verlag, New York, 1983.

2. Obradovic M., Ponnusamy S., Coefficient characterization for certain classes of univalent functions, Bull. Belg. Math. Soc. Simon Stevin 16 (2009), no. 2, 251-263.

3. Obradovic M., Ponnusamy S., Wirths K.J., Geometric studies on the class $\mathcal{U}(\lambda)$, Bull. Malays. Math. Sci. Soc. 39 (2016), no. 3, 1259-1284.

4. Obradovic M., Ponnusamy S., Wirths K.J., Logarithmic coefficients and a coefficient conjecture for univalent functions, Monatsh. Math. 185 (2018), no. 3, 489-501.

5. Obradovic M., Ponnusamy S., On the class $\mathcal{U}$, Proc. 21st Annual Conference of the Jammu Math. Soc. and a National Seminar on Analysis and its Application, 11-26, 2011.

6. Reade M.O., Silverman H., Todorov P.G., Classes of rational functions, Topics in complex analysis (Fairfield, Conn., 1983), 99-103, Contemp. Math., 38, Amer. Math. Soc., Providence, RI, 1985.

7. Thomas D.K., Tuneski N., Vasudevarao A., Univalent functions. A primer, De Gruyter Studies in Mathematics, 69. De Gruyter, Berlin, 2018.

Department of Mathematics, Faculty of Civil Engineering, University of Belgrade, Bulevar Kralja AleKsandra 73, 11000, Belgrade, Serbia

E-mail address: obrad@grf.bg.ac.rs

Department of Mathematics and Informatics, Faculty of Mechanical Engineering, Ss. Cyril and Methodius University in Skopje, Karpoš II B.B., 1000 Skopje, Republic of Macedonia.

E-mail address: nikola.tuneski@mf.edu.mk 